\title{
Design, 3D-printing, and characterisation of a low-cost, open-source centrifuge adaptor for separating large volume
} clinical blood samples

8 9

Md Ehtashamul Haque ${ }^{1}$, Linda Marriott ${ }^{1}$, Noman Naeem ${ }^{1}$, Taygan Henry ${ }^{1}$, Alvaro J. Conde ${ }^{1,2}$ and Maïwenn

$$
\text { Kersaudy-Kerhoas }{ }^{1} *
$$

${ }^{1}$ Institute of Biological Chemistry, Biophysics and Bioengineering, School of Engineering and Physical Sciences, Heriot-Watt University, EH14 4AS, Edinburgh, UK

${ }^{2}$ Micronit B.V., Colosseum 15, 7521 PV Enschede, Netherlands

*Corresponding author: Maïwenn Kersaudy-Kerhoas, m.kersaudy-kerhoas@hw.ac.uk

\begin{abstract}
Blood plasma separation is a prerequisite in numerous biomedical assays involving low abundance plasmaborne biomarkers and thus is the fundamental step before many bioanalytical steps. Conventionally, plasma separation is performed using high-capacity refrigerated centrifuges which have the advantage of handling large volume blood samples. These centrifuges are bulky, and prohibitively expensive for low-resource settings, with prices starting from $\$ 1,500$. Although commercial and existing open-source micro-centrifuges are relatively low-cost, they cannot handle large volume blood samples. Microfluidic blood plasma separation also has been adopted by many researchers to enable low-cost plasma separation, however, these systems still present yield and purity issues for extremely low abundance biomarker detection such as the detection of various fractions of circulating cell-free DNA. To overcome this, we customised the rotor of a commercially available microcentrifuge $\sim$ \$125) using fused filament fabrication to enable centrifugation of large clinical blood samples in resource poor-settings. Our designed adaptor (\$15) can hold two $9 \mathrm{~mL}$ S-Monovette tubes and maintain the same separation performance (yield, cell count, hemolysis, albumin levels) as the control benchtop refrigerated centrifuge. This low-cost open-source centrifugation system capable of processing clinical blood tubes could be valuable to low-funded laboratories or low-resource settings where centrifugation is required immediately after blood withdrawal for further testing.
\end{abstract}

Keywords: Centrifugation, Blood, cfDNA, open-source, microfluidics 


\section{Introduction}

A centrifuge is one of the most frequently used instruments in laboratory diagnostic and molecular biology laboratories, where it is employed to extract particles having different densities from a variety of mediums, using centrifugal forces. The primary uses of a centrifuge in a laboratory include the separation of plasma from whole blood for immunoassays or hematocrit analysis [1], the separation of pathogens and parasites in biological fluids [2], and DNA extraction preparation steps [3].

In particular, blood plasma separation is essential, and often the primary step in numerous biomedical assays involving low abundance target molecules, such as cell-free nucleic acids. Conventionally, this separation process is performed using high-capacity refrigerated centrifuges which are capable of dealing with a large volume (>5-50 mL) of blood samples. These laboratory centrifuges are bulky (120 kg), usually work with $20-40$ cm diameter rotors holding 20-100 sample vials, thus occupy a sizeable space in the laboratory space. Moreover, these large capacity centrifuges are expensive, (capital expenditure starts from $\$ 1,500$ ) and have high operational costs associated with them [4]. Although there are many micro-centrifuges available at relatively low cost (from $\$ 120$ ), they cannot handle volume blood samples above $1.5 \mathrm{~mL}$ and cannot deal with routinely used clinical blood tubes, such as S-Monovettes (Sarstedt, Germany). Furthermore, most of the available low-cost large capacity centrifuges cannot process the S-Monovettes, and thus introduces further preparation steps before the centrifugation. Therefore, a low-cost centrifugation system capable of handling SMonovette tubes would be beneficial to many low-funded laboratories in developing countries where centrifugation is required immediately after the blood withdrawal for further testing.

To reduce the cost of centrifugation, a number of so-called "frugal" solutions have emerged, as part of the opensource and open-science movement [5]-[7]. A completely 3D printed centrifuge requiring no electricity [1], a portable low-cost centrifuge [8], a human-powered paper or 3D-printed centrifuge [9], a device based on the fidget-spinner toy [10], and a centrifuge adapter for a power tool [11] have been proposed, amongst other solutions. However, most of these devices are only able to handle low volume samples typically from a few microliters to $1.5 \mathrm{~mL}$, and none of them can use clinical blood collection tubes (such as S-Monovette) directly after the blood withdrawal. Figure 1.A shows the processing volume of available commercial and open-source academic microcentrifuges against their price. Figure 1.B illustrates the size difference between the blood handling tubes commonly used in microcentrifuges and the clinical S-Monovette collection tube. We have identified the need for a low-cost, yet automated, approach to the centrifugation of larger sample volumes. Such a device could facilitate access to relevant laboratory tools and expand scientific or medical research and practice.

In this work, we propose a novel approach to significantly reduce the cost of S-Monovette centrifugation by customising the rotor of a commercially available microcentrifuge (SciSpin MINI Microfuge, model: SQ-6050) using low-cost additive manufacturing (3D printing) (Figure 1.C). 3D printing has emerged in recent years as a convenient method for the development of cost-effective and open-source scientific and diagnostic tools [6], [12]-[14]. We have made this solution open-source and all the manufacturing details are included for others to replicate this work. This customization procedure could be beneficial to scientists in a low-resource environment [5]-[7], to scientists in need of a mobile centrifugation solution, or to educators. This adapted centrifuge is able to hold two standard $9 \mathrm{~mL}$ S-Monovette tubes and maintain almost the same separation efficiency as a 

aerodynamic drag force) on the behaviour and performance of the 3D printed rotor using plasma yield, blood cell counts, cfDNA and albumin levels for biological characterisation.

A

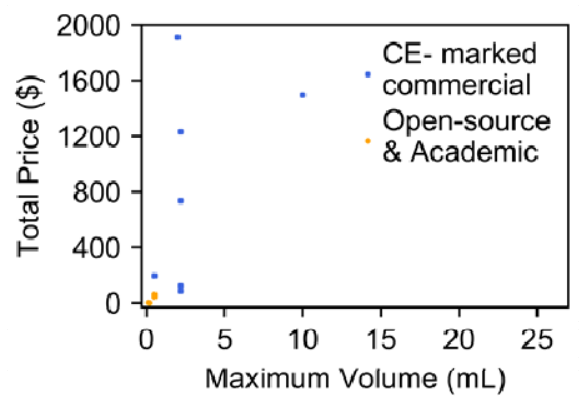

B

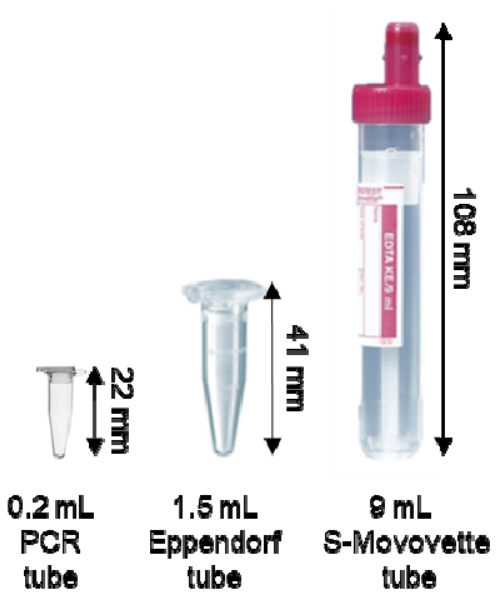

\section{C}

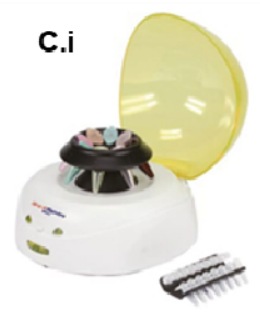

Mini-centrifuge $\operatorname{Max} 8 \times 1.5 \mathrm{~mL}$

I

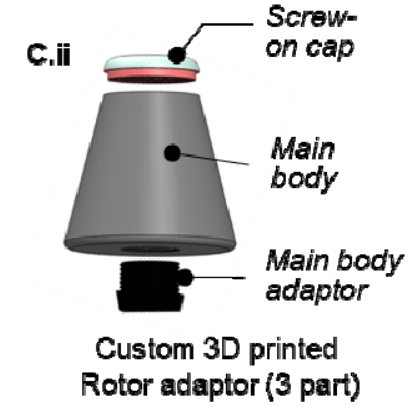

Rotor adaptor (3 part)

Figure 1: A) Sample processing volume vs price of available commercial and open source academic microcentrifuges, $\boldsymbol{B})$ Illustration of the different sized tubes used with the available microcentrifuges along with $S$-Monovette tube used in this project, $C$ ) Adaptor concept: C.i) Commercial SciSpin MINI Microfuge, model: SQ-6050 with its original rotors $\boldsymbol{C . i i ) ~ C A D ~ s c h e m a t i c ~ o f ~ t h e ~ d e s i g n e d ~ t h r e e - p a r t ~ r o t o r ~ a d a p t o r ~ C . i i i ) ~ F i n a l ~ 3 D ~}$ printed rotor adaptor mounted on the commercial microcentrifuge base $\boldsymbol{C}$.iv) Top cover removed from the adaptor, showing inside part of the rotor adaptor that holds two standard $9 m L S$-Monovette tubes.

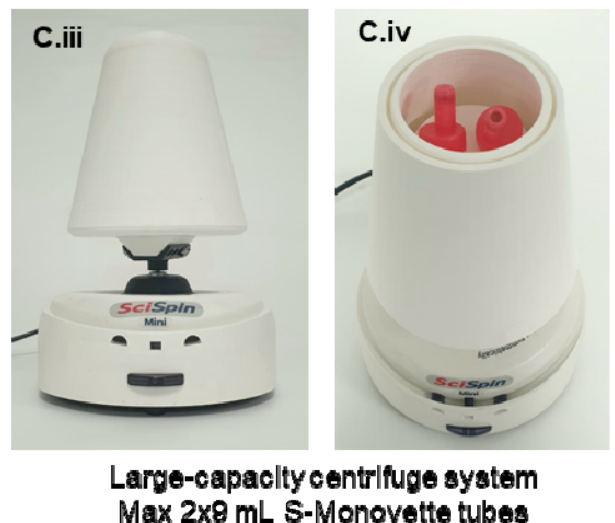

\section{Material and methods}

2.1 Theoretical background and design consideration

Centrifugation is a way to increase the gravitational field magnitude by spinning a sample around an axis which creates a relative centrifugal force ( $\mathrm{RCF}$, also called the "g" force) capable of pulling cells and other particles to the furthest position from the centre of rotation. The RCF is proportional to the square of the rotor speed and the radial distance and can be calculated using the following equation: 

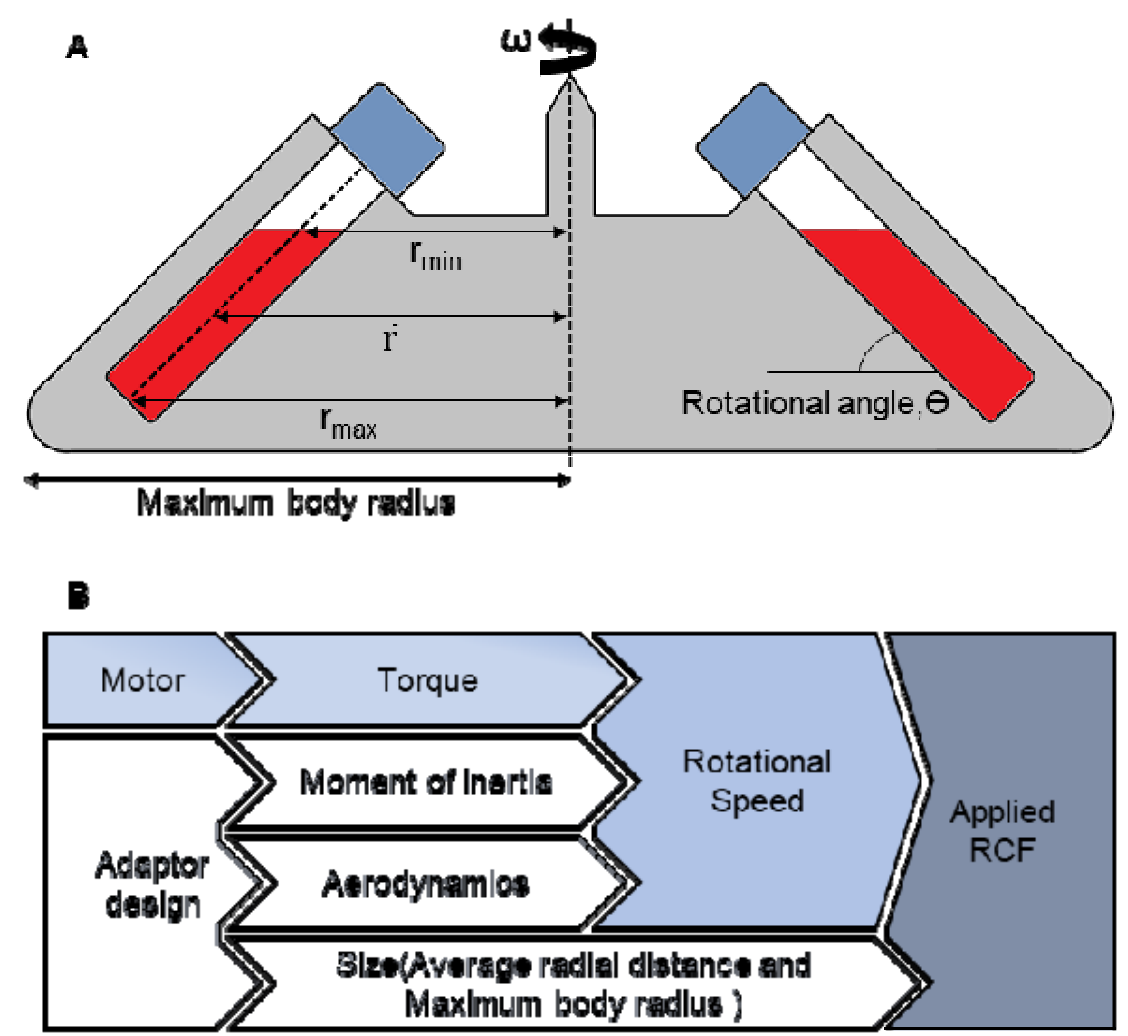

$91 \quad$ Figure 2: (A) Schematic illustration of the tubes on a fixed-angle adaptor and the radial dimensions $(\boldsymbol{B})$ The influential parameters on the applied RCF on the sample in the tube mounted on the adaptor

93 Although it seems from equation (1) that the RCF should increase with an increment in speed and the distance of the sample from the axis, other parameters must be considered to obtain the highest RCF. These parameters include the moment of inertia, torque, and aerodynamics (Figure 2.B). As the original rotor is going to be replaced by the customised one, to meet this additional load demand, the torque of the motor will increase, which will force the speed to go down because of the inverse relationship between the torque and speed. As the RCF increases with the square of the rotor speed, halving the speed will result in a 4-fold decrement in RCF which will result in poor separation efficiency. The additional mass and larger size of the rotor adaptor to accommodate the S-Monovette tubes lead to a decrease in the speed because of the larger moment of inertia and higher aerodynamic drag force. Therefore, although the increment of radial distance increases the RCF, it can also decrease the rotational speed because of the increased moment of inertia and aerodynamic drag force. In this trade-off between speed and radial distance, the speed has been given priority because of its squared relationship with the RCF and every effort has been made to keep the design as small and light as possible. In addition to these discussed parameters, the rotational angle also plays a vital role in the separation process where most compact pellet after the separation usually forms with a higher rotational angle and therefore $45^{\circ}$ fixed angled rotors are most in commercial centrifuges that uses fixed-angle rotors. It should be noted that, among the two types of rotors, fixed-angle and swing-bucket rotors, the former one has many advantages like lower exposure to stress, higher RCF and not having any moving hanging parts; hence, adopted in our project. The effect of different angled rotors has been studied in different designs which will be discussed in the next sections. 


\subsection{Centrifuge hardware}

114 The commercial microcentrifuge (SciSpin MINI Microfuge, model: SQ-6050) used in this project is widely 115 available on mainstream purchasing platforms. To the best of our knowledge, this is the lowest CE-marked cost

116 microcentrifuge on the market at the time of writing. It also has a higher rotational speed (7000 RPM), is 117 lightweight and is more compact compared to other available devices. The full technical specifications of the 118 microcentrifuge are provided in Supplementary Table 1. A commercial benchtop refrigerated centrifuge 119 (Allegra X-12R, Beckman Coulter) with a swinging bucket, (Modular Disk Adapters for Tubes (SX4750)) was 120 used as the benchmark. The benchmark centrifuge was always run with an RCF of $3273 \times \mathrm{g}$ (3750 RPM). The 121 RCF for the benchmark was chosen as the maximum speed allowed on a swinging bucket configuration and 122 close to the average first spin values used in cfDNA studies [15]. The maximum speed for the commercial 123 Allegra centrifuge is respectively 3750 and 10,200 RPM for swinging bucket and fixed angle, (equivalent RCF 124 respectively 3720 and $11,400 \times \mathrm{g}$ ) The second spin protocol applied in plasma quality measurements was $12512,000 \times \mathrm{g}$ RCF for 10 minutes with a commercial high-speed microcentrifuge (5417 R, Eppendorf), also based 126 on cfDNA studies [15].

\section{$127 \quad 2.3 \quad 3 \mathrm{D}$ printed adaptor fabrication}

128 All 3D printed rotor adaptors presented here were designed using 3D modelling CAD (SolidWorks, 2018) and 129 fabricated using Fused Deposition Modelling (FDM) technology. The Anycubic i3 Mega (Anycubic, Shenzhen, 130 China) 3D printer was employed for printing. Polylactic Acid (PLA) (Verbatim 1.75mm clear PLA, brand) was 131 used to print the rotor. The technical characteristic of the printer is provided in Supplementary Table 2. The 132 objects were sliced with Ultimaker Cura 4.4 [16] using the standard settings summarized in Supplementary 133 Table 3. All the design files are provided in .stl format in the online repository FigShare 134 (https://doi.org/10.6084/m9.figshare.16762444.v2 ).

$135 \quad 2.4 \quad$ Simulation of critical speed

136 Any rotating system tends to vibrate in the absence of a driving force at certain frequencies called natural 137 frequencies. When the frequency of the rotational speed matches with the natural frequencies of the system, 138 there will be a resonance and the system will vibrate at that frequency. This natural frequency matching speed is 139 commonly known as the critical speed of the system. The vibration of the rotating system can impose high shear 140 stress on the blood cells and could potentially damage the cells, leading to hemolysis, the destruction of red 141 blood cells, and erroneous analytical results [17]. Therefore, it is important to minimize the critical speed to 142 ensure the minimum shear stress on blood cells. Industrial centrifuge systems operate below and above critical 143 speeds and the critical speeds are controlled via damping in rotor shaft connection [18]. In this project, the 144 critical speed of different designs was predicted in-silico using Ansys workbench modelling (2021 R1, student 145 edition) with the purpose of minimising the model critical speed. PLA material was assigned during modelling 146 and the threads present in the original design were removed to facilitate the meshing. Fine meshing was the 147 highest possible meshing setting available with the Ansys version used in this study. Initially, Modal analysis 148 was used to calculate the natural frequencies and fifteen rotational speeds were used between 0-7000 RPM with 149500 RPM intervals during the process. Finally, Campbell diagrams were used to calculate the critical speed from 150 the modal analysis. 


\subsection{Deflection measurement}

The deflections of each 3D printed adaptor were measured from video recordings using a code written in the Python OpenCV module. Three video recordings of each adaptor were taken, with a duration of 4 minutes (At 0 sec, Motor mains and video start; at $2 \mathrm{~min}$, Motor mains stop; at $4 \mathrm{~min}$, video stop). Prior to the deflection measurement, sizes of the video files were reduced using an online video converter (https://ezgif.com/). In the first module of the python code, the edge position of the adaptor was detected and transformed in pixel units for each frame of the video. Then these detected positions were compared with the initial reference and deflections in the pixel unit were incorporated into a final deflection matrix. Finally, the values of the deflection matrix were saved to an MS Excel file along with their corresponding pixel numbers. The code saved the screenshot of the processed video images from which a pixel per mm value was calculated from a known distance. This calculated pixel per mm value was then compared to the saved deflections values in the MS Excel file which provided the absolute deflection in $\mathrm{mm}$ for each design. The Python code is provided as a supplementary file along with an example video of Design C. All video recordings are available from the FigShare repository https://doi.org/10.6084/m9.figshare.16762444.v2

\subsection{Sample material}

Human blood samples were obtained under local ethical approval from the Scottish National Blood Transfusion Service (contract \#18 06) and according to the Declaration of Helsinki. Samples were kept refrigerated (2-8 degrees) before their use. Blood samples were ordered from the same group (O positive) and pooled. Upon arrival, they were mixed gently to save the RBCs from getting damaged via excessive shear stress. Prior to each experiment, $9 \mathrm{~mL}$ blood samples (from the same pool) were poured into $9 \mathrm{~mL}$ S-Monovette opened syringes using S1 Pipet Filler (Thermo Scientific) which enabled fatigue-free pipetting. The larger diameter of the pipette tip ( 0.9 mm) ensured lower stress exerted during blood aspiration.

\subsection{ImageJ analysis on yield calculation}

Prior to the experiments, all the S-Monovette tubes were marked to indicate the area required for a $1 \mathrm{~mL}$ volume of plasma. After each experiment, photographs of the tubes in a fixed custom set-up were captured with a mobile camera (Samsung Galaxy Note9). Thereafter, the captured images were processed using ImageJ software to measure the yield of the separated plasma. During the analysis, the previously marked area was selected as the scale. The plasma volume separated after centrifugation was calculated from the position of the plasma limit. Finally, the yield was calculated by comparing this separated plasma volume with the total available plasma in the sample, known from the hematocrit (Hct) measurement (See section 2.8).

\section{$2.8 \quad$ Blood cell counts}

RBCs and platelets count, hematocrit (Hct), and hemoglobin (Hgb) of all the pre-and post-centrifuged blood and plasma samples were measured by a hematology analyzer (Sysmex XP-300, Sysmex Corporation, Japan). These measurements were used to compare the separation efficiency and purity of different designs.

\subsection{Characterisation of hemolysis via spectrophotometry}

A centrifuge operates at a high centrifugal force and may exert a shear rate on the RBCs resulting in hemolysis. The reference for hemolysis rate during storage provided by the American Society for Clinical Pathology is $2 \%$ or less [19], the Council of Europe guidelines recommend not to exceed 0.8\% [20] and the US FDA 1\%. While 
189 these values provide a useful guide to interpret the effect of centrifugation on blood samples, it should be noted

190 that they refer to hemolysis in blood and blood components intended for transfusion or for further manufacture,

191 not in-vitro diagnostics. During hemolysis, RBCs release their Hgb content on the sample. Free Hgb

192 measurements in plasma provide an estimation of the overall hemolysis of a sample. In order to evaluate the

193 hemolysis generated during the centrifugation with different devices, Hgb concentration on the plasma was

194 measured using the spectrophotometric Cripps method at 560, 576 and $592 \mathrm{~nm}$ wavelengths [21]. The

195 percentage of hemolysis was estimated with equation (2 [22]:

$$
\text { Hemolysis Percentage }(\%)=\frac{(100-H C T) \times \text { Free HGb }}{\text { Total Hgb }}
$$

where Hct and Total Hgb represents the total hematocrit and total Hgb content of the initial blood sample and free $\mathrm{Hgb}$ is the estimation of Hgb concentration using the Cripps method in an undiluted plasma sample. In this method, background absorption from other proteins, such as bilirubin, is automatically mitigated by the fractional absorbance between $576 \mathrm{~nm}$ and at $560 \mathrm{~nm}$ and $592 \mathrm{~nm}$ wavelengths. In order to quantify the absolute free Hgb level in our samples, a standard curve was obtained by diluting human Hgb powder (Sigma-Aldrich, USA) in human plasma (Cambridge Bioscience, UK) to make samples of 1, 0.5, 0.1, 0.05, 0.01, and 0.005 $\mathrm{mg} / \mathrm{dL}$. The absorbance of the plasma samples extracted after centrifugation of different devices along with the standard samples made from human plasma was measured with the 96 well plate reader (POLARstar Omega, BMG Labtech).

\subsection{0 cfDNA extraction}

To assess cfDNA levels in several designs and controls, total cfDNA was extracted from $3 \mathrm{~mL}$ of separated plasma using the QIAamp Circulating Nucleic Acid kit (QIAGEN) following manufacturer instructions. Extracted cfDNA samples were frozen until use. Real-time quantitative PCR was performed using 2x Power SYBR® Green PCR Master Mix (Thermo Fisher Scientific) to amplify 90 bp target with LINE primers (final concentration $200 \mathrm{nM}$ ): forward 5'-TGC CGC AAT AAA CAT ACG TG -3' and reverse 5'-GAC CCA GCC ATC CCA TTA C-3'[23]. A standard curve was created using a series of 5 dilutions of Human Genomic DNA. Thermal cycling conditions involved a 10 -minute cycle at $95^{\circ} \mathrm{C}$ followed by 40 cycles with 15 seconds at $95^{\circ} \mathrm{C}$ and 60 seconds at $60^{\circ} \mathrm{C}$. Samples were amplified in triplicates using Applied Biosystems StepOnePlus ${ }^{\mathrm{TM}}$ RealTime PCR System (Applied Biosystems). A melting curve was performed as a control measure for non-specific amplification. Absolute amounts in each sample were obtained from the standard curve.

\subsection{Protein load}

Bromocresol Purple (BCP) Albumin Assay Kit (Sigma-Aldrich, Merck, Germany) performed as per manufacturer's instructions with plasma samples diluted 5-fold in ultrapure water. The kit utilizes bromocresol purple, which forms a colored complex specifically with albumin. The intensity of the colour, which is directly proportional to the albumin concentration in the sample, was measured at $610 \mathrm{~nm}$ with a 96 well plate reader (POLARstar Omega, BMG Labtech). 


\section{$222 \quad 2.12$ Statistical Analysis}

223 Statistical significance was determined by an unpaired parametric Student t-test. Unless specified, the p-value

224 significance threshold was set at 0.05 . When reporting on statistical significance symbols ' $n s$ ' is used to indicate

225 non-significance $(\mathrm{P}>0.05)$, while *, **, $* * *$ denotes $\mathrm{p}<0.05, \mathrm{p}<0.01, \mathrm{p}<0.001$ as per conventional practice.

$226 \quad 2.13$ Safety Notice

227 To accommodate the large rotor adaptor, the original safety lid of the mini-centrifuge was removed. During the

228 development phase, other safety measures were put into place in case of an adverse event (e.g., accidental

229 detachment of rotor adaptor). Firstly, the base of the microcentrifuge was fixed with a $5 \mathrm{~mm}$ Perspex sheet with

230 recessed screws. Secondly, all the experiments were performed under a custom $6 \mathrm{~mm}$ thick Perspex safety hood.

231 All the designs went through a 30-minute continuous runtime without incident. None of the designs showed any

232 overheating, rotor displacement/detachment. The motor power load was measured on A-E0 designs on a Bench

233 Digital Multimeter (Keithley DMM6500, Tektronix, Beaverton, OR, USA) (Supplementary Info Table 4) and

234 found to operate well below the indicated power rating of the original instrument at full speed. Peak power loads

235 within $10 \%$ of the original power rating were observed at the beginning of each cycle. Motor power load

236 measurements are available on Figshare at https://doi.org/10.6084/m9.figshare.16762444.v2. While we are

237 confident the adapted rotor can be safely operated, we recommend additional precautions, such as (i) performing

238 a thorough visual inspection of the printed parts to ensure they have no defects (ii) ensuring the correct balance

239 of the tubes prior to spin, and (iii) operating the instrument within an appropriate enclosure (we recommend at

$240 \quad$ least $5 \mathrm{~mm}$ thick solid polycarbonate).

\section{Results and discussion}

\subsection{Design progression}

243 A total of thirteen designs were manufactured and tested. Each design is presented in Table 1 alongside physical

244 parameters. All the designs are capable of holding two $9 \mathrm{~mL} \mathrm{~S}$-Monovette tubes. Parameters such as the size of

245 the device, weight, radial distance, aerodynamic drag force around the device and the rotational angle were

246 explored. Design A and B are the preliminary rotor designs planned without aerodynamic drag force

247 considerations where we varied the angles and radial distance of the two designs while keeping the weight

248 constant around $60 \mathrm{~g}$ to observe the radial distance effect on the RCF.

249 Table 1: Evolution of design and basic characterisation 


\begin{tabular}{|c|c|c|c|c|c|c|c|c|}
\hline Design & Schematic & $\begin{array}{c}\text { Cross } \\
\text { section }\end{array}$ & $\begin{array}{c}\text { Tube } \\
\text { Angle } \\
\left({ }^{\circ}\right)\end{array}$ & $\begin{array}{c}\text { Rotor } \\
\text { mass } \\
(\mathrm{g})\end{array}$ & $\begin{array}{c}\text { Average } \\
\text { radial } \\
\text { distance, } \\
\bar{r}(\mathrm{~cm})\end{array}$ & $\begin{array}{l}\text { Max. } \\
\text { body } \\
\text { radius } \\
(\mathrm{cm})\end{array}$ & $\begin{array}{l}\text { Rotat- } \\
\text { ional } \\
\text { speed } \\
\text { (RPM) }\end{array}$ & $\begin{array}{c}\text { Applied } \\
\text { RCF } \\
(\times g)\end{array}$ \\
\hline \multirow{5}{*}{ A } & & \multirow{5}{*}{ N/A } & 30 & 60.7 & 7.677 & 10.1875 & 1708 & 250 \\
\hline & & & 37.5 & 59.9 & 8.337 & 10.825 & 1607 & 241 \\
\hline & & & \multirow{3}{*}{45} & 60.5 & 7.979 & 10.55 & 1719 & 264 \\
\hline & & & & 60.33 & 8.979 & 11.55 & 1524 & 233 \\
\hline & & & & 60.8 & 9.979 & 12.55 & 1345 & 202 \\
\hline \multirow{3}{*}{$\mathbf{B}$} & & \multirow{3}{*}{ N/A } & 30 & 59.3 & 6.204 & 8.57 & 2101 & 307 \\
\hline & & & 37.5 & 59.2 & 6.598 & 9.091 & 1890 & 293 \\
\hline & & & 45 & 59.1 & 7.135 & 8.65 & 1809 & 291 \\
\hline C & & & 45 & 364.8 & 4.459 & 8.25 & 4160 & 884 \\
\hline D & & & 45 & 384 & 3.835 & 8.275 & 4505 & 871 \\
\hline EO & & & 25 & 297.3 & 2.278 & 6.25 & 6392 & 1043 \\
\hline E2 & & & 25 & 338.6 & 2.094 & 6.05 & 6725 & 1060 \\
\hline E5 & & & 25 & 272.8 & 2.088 & 5.75 & 6884 & 1097 \\
\hline
\end{tabular}

251 Although the radial distance of design $\mathrm{A}$ is higher than that of design $\mathrm{B}$, the RCF of Design A is smaller because

252 of the higher speed obtained by design B. A close inspection of angle variations also shows similar phenomena.

253 The radial distance of higher angled designs is slightly higher than the smaller ones. As an example, the radial

254 distance of design $\mathrm{B}-45^{\circ}$ is $0.931 \mathrm{~mm}$ higher than that of $\mathrm{B}-30^{\circ}$ design and the RCF value is $16 \times \mathrm{g}$ lower. This can be explained by the aerodynamic drag force acting during the rotation which is directly related to the drag coefficient (depends on the shape of the body), the frontal area and the square of the rotating velocity [24]. The frontal area of design A and B can be compared to a flat plate that is highly resistive to the surrounding air and have a high drag coefficient of 1.17 to 1.98 [25], [26]. With a higher radial distance or angle, the frontal area becomes even bigger and minimize the speed and RCF value. It is also noteworthy that the maximum achievable speed of designs A and B is much smaller than the rated speed of the motor because of their high air resistive flat plate shape. Therefore, although these initial arbitrary shaped designs have some advantages such as the low material amount and low material cost, and fast printing time ( $<2$ hours), the achieved speed and $\mathrm{RCF}$ are not capable of highly efficient centrifugal separation. 
In the subsequent designs (C-E), the aerodynamic drag force was considered and a truncated cone shape with a drag coefficient of about 0.05 to 0.5 was designed [25], [26]. A schematic diagram of the aerodynamic drag force around the designs A-B and C-E is provided in Figure 3.A. The speed of the truncated conical shape devices was over two folds that of the primary designs A-B. The relationship between the radial distance and speed for all designs is displayed Figure 3.B.
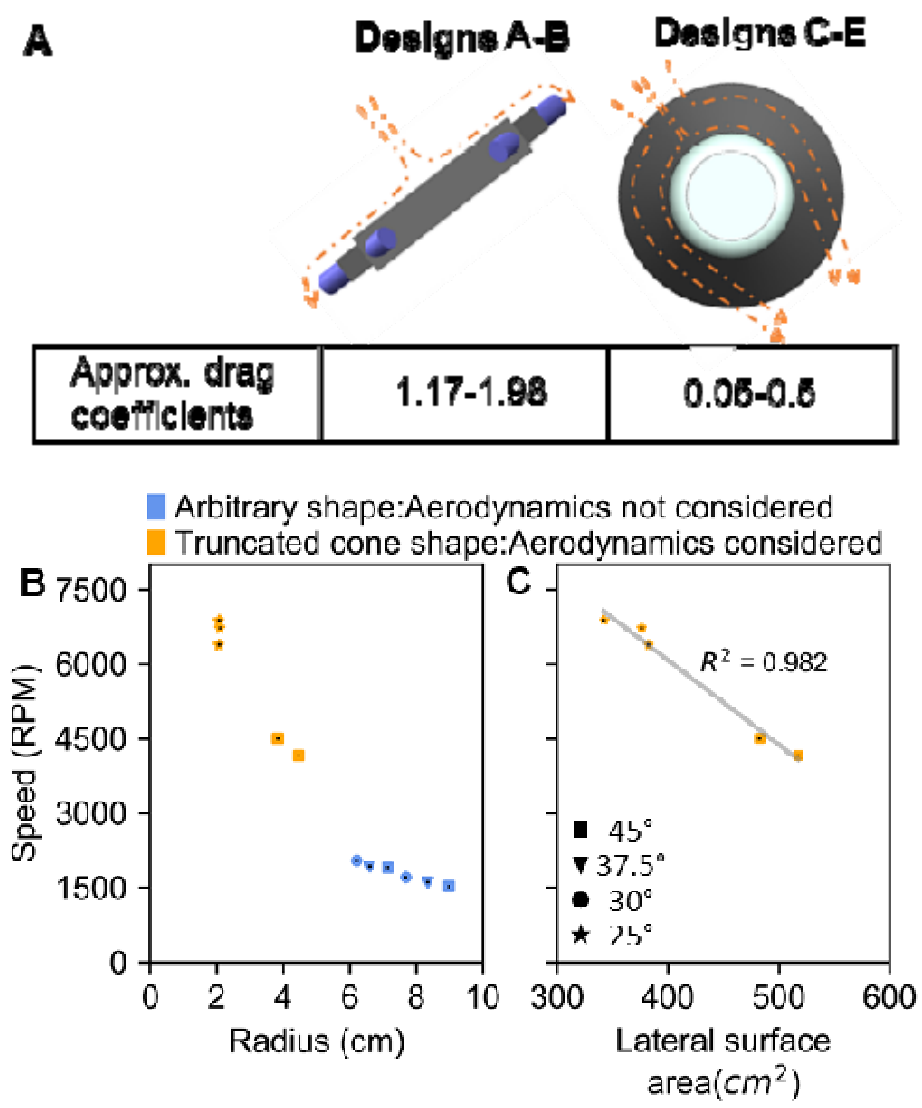

Figure 3:Influence of design shapes and sizes on speed (A) Schematic diagram showing an arbitrary shape design provides higher aerodynamic drag force than the truncated cone shape design by resisting much of the surrounding airflow. For simplicity, airflow is showed only in one direction instead of all sides. (B) Radial distance of all designs vs their rotational speed $(\boldsymbol{C})$ Lateral surface area of the truncated cone-shaped designs $v$ s their rotational speed. NB: Both the panel $B$ and $C$ share the same legend.

After achieving a higher speed (4160 RPM) with the original truncated cone shape design C, speed was further increased with the later designs D-E by decreasing the lateral surface area (Figure 3.C). Design with a smaller lateral surface area provided the least air resistance during the rotation which increased the speed of these designs. The final design E5, achieved a rotational speed of 6884 RPM, almost the rated speed of the original mini-centrifuge, and a RCF value of around 1100×g. It can be noted that the final three designs (E0, E2 and E5) all possess a $25^{\circ}$ rotational angle. Although a higher angle is recommended to ensure the most compact pellet, the trade-off has to be made to increase the speed and RCF of the designs.

\subsection{Truncated cone shape design and vibrational analysis}

In the previous section, we discussed that by considering the aerodynamic drag force with the first truncated cone shape design, Design $\mathrm{C}$, the rotational speed was increased almost 2-fold compared to design B where a 
284 lower speed has been observed because of its flat plat like structure which increased the aerodynamic drag force

285 of the body. However, with higher speeds, vibrations were observed during the ramping up or slowing down of

286 the rotation. In order to understand the nature of these vibrations and reduce them, we used video analysis to

287 measure their amplitude and duration (see Material and Methods section). Figure 4.A illustrates this process on

288 Design C. Figure 4.i and 4.Aii show snapshots of the recorded video before, and after, the edge detection with

289 the Python OpenCV code. Figure 4.A.iii shows the deflection measurements obtained from one of the Design C

290 videos. A deflection around $4 \mathrm{~mm}$ was observed both in the accelerating and decelerating phase at nearly 800

291 RPM, which by definition constitutes the critical speed of this design. These vibrations at high speed could

292 damage the RBCs because of high stress, hence the primary target was to lower the critical speed of the later

293 designs while ensuring higher rotational speed. To serve this purpose, the lateral surface area of the

294 abovementioned designed devices has been decreased to boost the rotational speed while Ansys workbench

295 modelling helped to predict the critical speed before printing the devices. 


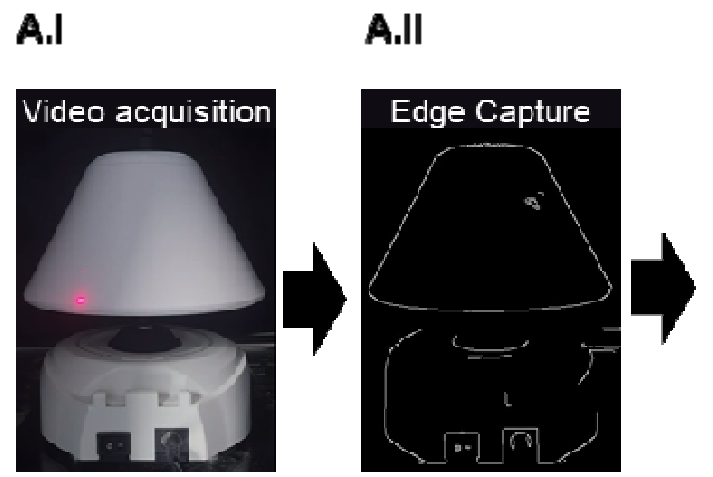

A.III

B.I B.II

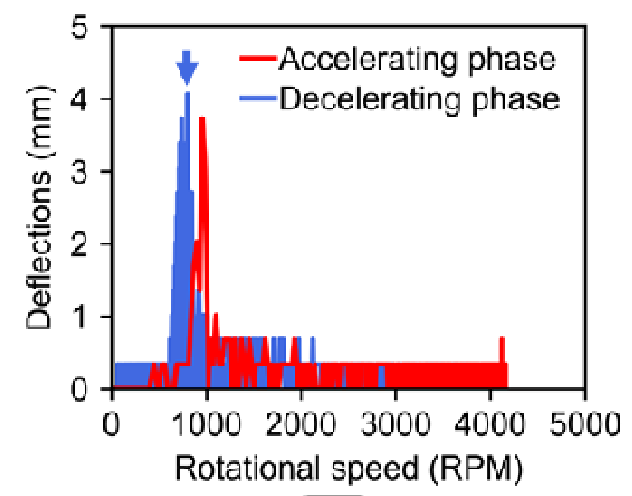

B.

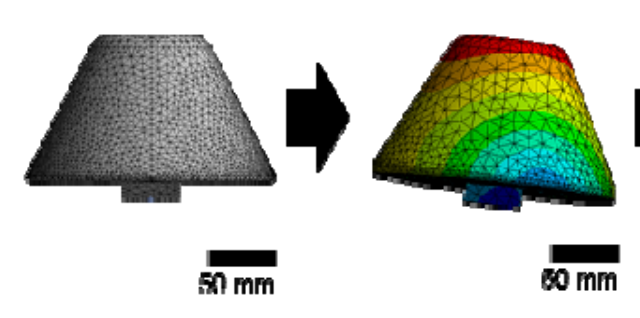

B.III
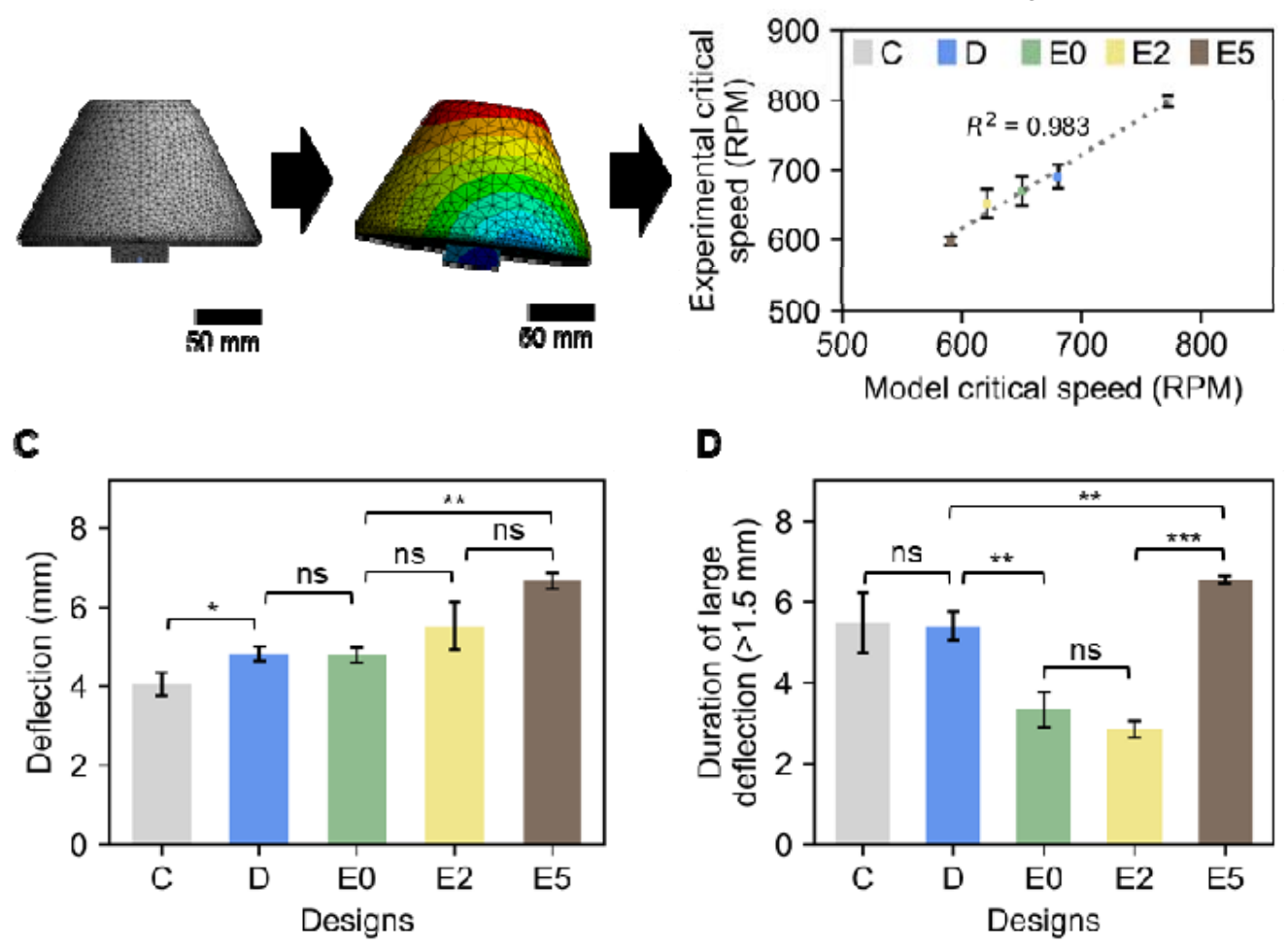

D

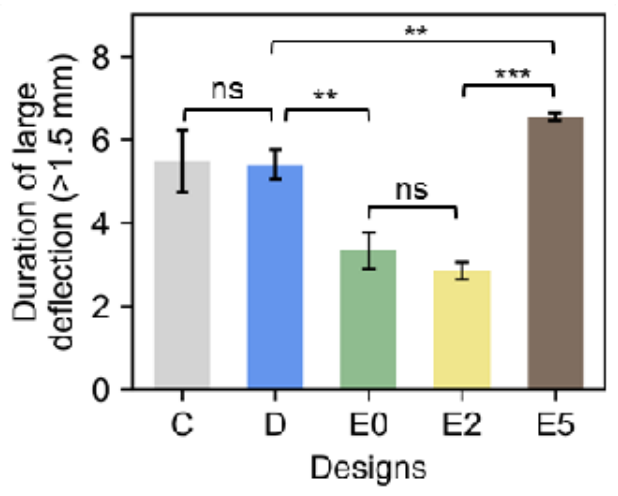

Figure 4: (A) Deflection measurement from the recorded video of conical Design C. A.i) Snapshot of one of the video recordings of Design C A.ii) Edge capturing from one of Design C videos using Canny, an edge detection operator in Python OpenCV module. Further details about the deflection measurements are available in Supplementary Figure S1 A.iii) Measured deflection of both acceleration and decelerating phase from the recorded video using python OpenCV module for Design C. All recordings are available from Supplementary Figure S2 (B) Critical speed measurement in Ansys workbench B.i) Meshing of Design C (Nodes: 6749, Elements: 39228) C B.ii) Mode shape during natural frequency B.iii) Simulated (model) vs experimental critical speed of each conical shape design showing a good agreement between them with low percentage difference (1$5 \%)(C)$ Measured deflection of each conical shape design showing all designs experiencing deflection from 4 to $7 \mathrm{~mm}(\boldsymbol{D})$ Duration of deflection higher than $1.5 \mathrm{~mm}$ was decreasing the lowering of critical speed except design E5 which experienced higher deflection for longer times.

The fine meshing of design $\mathrm{C}$ and the mode shape during the deflection of the device can be observed from Figure 4.B.i and ii, for illustration. The Ansys model correctly predicted the critical speed of each model with high accuracy (model vs experimental measurement values in Figure 4.B.iii, $\mathrm{R}^{2}=0.983$ ). The maximum deflection of each design at their critical speed can be found in Figure 4.C. The final design, E5 with the lowest 
311 critical speed of around 600 RPM provided a maximum deflection of around $7 \mathrm{~mm}$ whereas for the other

312 designs the average deflection was limited within 4 to $5 \mathrm{~mm}$. These deflections can be interpreted as impulsive

313 vibrations which might exert excessive shear stress on the RBCs and cause hemolysis [27], [28]. Therefore, the

314 final degree of hemolysis depends on the shear stress exerted on RBCs and the exposure time of the impulse

315 vibration [29]. Figure 4.D illustrates the duration of deflection higher than $1.5 \mathrm{~mm}$. The duration of large

316 deflections was minimised from C to E2. However, design E5 appeared to be providing maximum deflection for

317 about 7 seconds. Therefore, despite having the highest rotational speed and lowest critical speed, we predicted

318 that E5 would have a low separation efficiency because of the amplitude and the duration of these impulsive

319 vibrations.

\section{$320 \quad 3.3 \quad$ Plasma yield}

321 The separation performance of a centrifuge can be described by the separation yield. The separation yield 322 indicates how much volume of plasma the centrifuge can separate from the total volume of available plasma. To determine the plasma separation yield, $9 \mathrm{~mL}$ of pooled blood samples were centrifuged with each conical design (C, D, E0-2-5) and the full-scale centrifuge control for 3, 6 and 10 minutes and the separated plasma volume and full blood count were measured. Figure 5 shows photographs (panel A) and quantitative results of plasma yield in the initial sample and samples after 3,6, and 10 minutes centrifugation on the adapted centrifuge and the control.
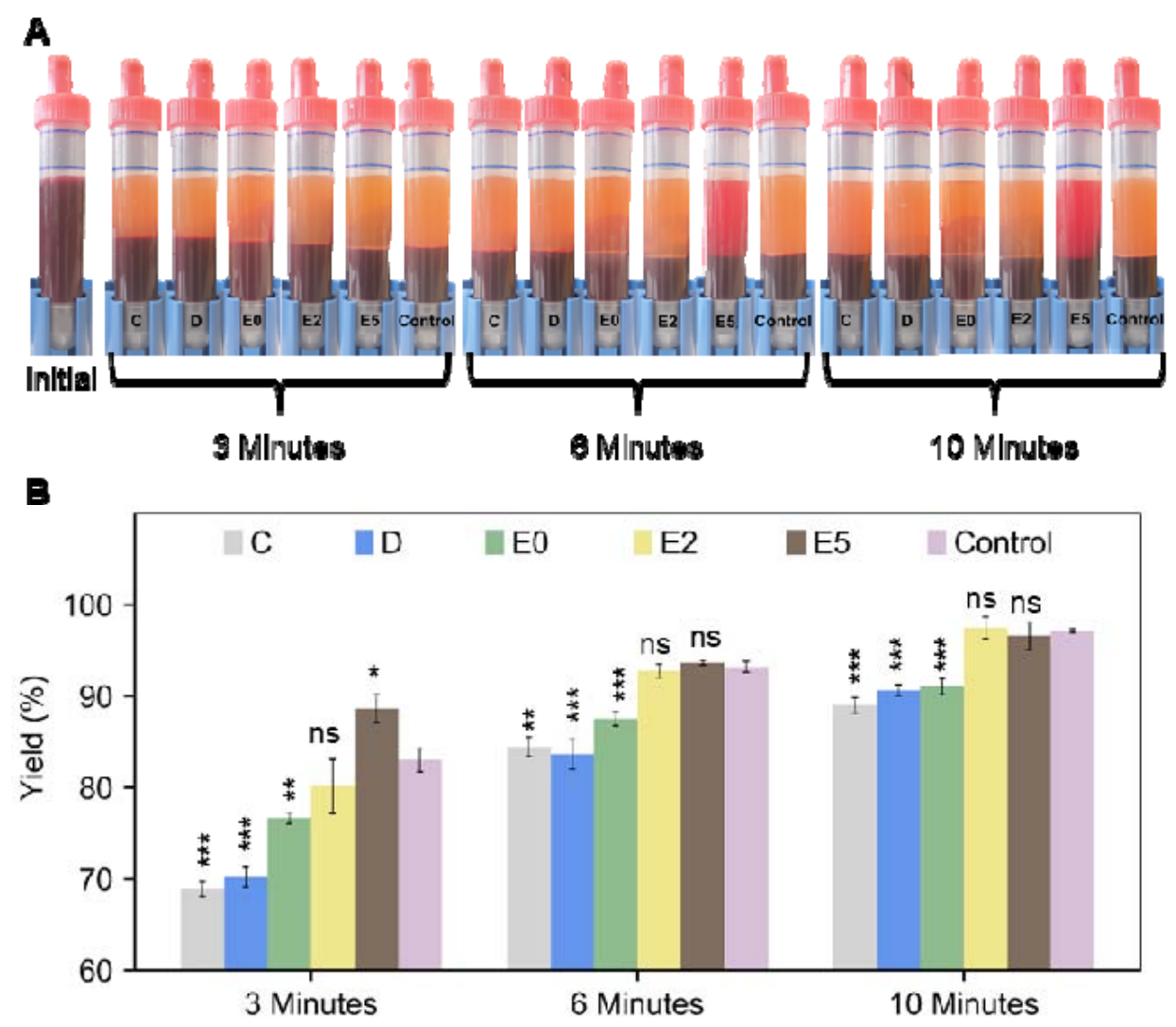

Figure 5: (A) Images of the initial and post centrifugated (3,6,10 minutes) S-Monovette tubes showing that larger centrifugation times resulted in a higher volume of plasma separated from $9 \mathrm{~mL}$ sample. (B) Measured 
unpaired t-test between each column and the control. Design E2 and E5 were able to separate the same amount of plasma as the control at 6 and 10 minutes with no statistically significant difference.

It is worth noting that most of the plasma gets separated within 3 minutes of centrifugation (minimum separation $\sim 70 \%$ for Design C) and the separation volume further increases at 6 and 10 minutes. The single most striking observation is the decreased separation performance of Design E5 at 6 and 10 minutes. Device E5 shows much greater performance after 3 minutes centrifugation ( 90\% yield) compared to the control. However, during 6and 10-minutes separation instances, the design failed to maintain this higher performance where it was unable to separate all RBCs present in the separated plasma. To facilitate the discussion here, it needs to be clarified that this reddish colour of plasma at 6 and 10 minutes was exclusively because of the nonseparated red blood cells present in plasma, not due to any RBC damage which was quantified and will be described later. The internal shape of design E5 might be a reason behind this poor separation efficiency. This design is comparatively the smallest of all the other designs and to accommodate this size, the sidewall was brought very close to the placed tube inside and furthermore, the tube was completely encased against the sidewall, unlike the other design where the tubes are hanging freely, away from sidewalls. In the previous section, we noticed a high deflection and duration of deflection with design E5 which we suspect might cause the sidewall to touch the SMonovette tubes randomly at several instances and hinder the separation efficiency. With device E2, it was possible to achieve almost $80 \%$ yield within 3 minutes which was almost equivalent to the performance of the control. The comparative yield of design E2, E5 (approximatively 95\%) are not significantly different from the control after 10 minutes of centrifugation.

As discussed previously, the overarching goal in this study was to customize a low-cost microcentrifuge in such a way that it could handle a large $9 \mathrm{~mL}$ clinical sample volume directly after the blood withdrawal without any extra blood handling steps. At the same time, the device should ensure at least similar separation performances as a benchtop refrigerated centrifuge. We, and others in the field, have previously demonstrated microfluidic solutions as an alternative to traditional centrifugation [30]-[33]. However, we have concluded that for extremely low biomarker detection such as the detection of various fractions of circulating DNA, microfluidic approaches are not viable because of their low separation yield and the difficulty to handle the large volume of the viscous whole blood sample. The main barrier in the microfluidic approach is the cell-cell interaction between red blood cells. When high volume fractional blood is flowed at a high flow rate, the cell interaction between a large number of RBCs in the microfluidic channel increases, which inhibits the cell-free layer formation and other deterministic effects, thus reducing the separation performance [34]. As a result, the use of high-volume fractional blood in the above comparative high-throughput studies has resulted in much lower separation yields. Comparatively, we can see in this study, design E0 and E2 managed to secure more than $95 \%$ of the available plasma within 10 minutes of centrifugation. This high plasma yield is a valuable factor in lowlevel biomarker detection.

\subsection{Plasma quality: Residual blood cell count}

Firstly, the total RBC count was measured each time the blood sample was remixed following centrifugation to investigate the integrity of the blood sample after centrifugation. Figure 6.A presents the pre-and postcentrifuged whole blood RBC count at 3,6 and 10 minutes. It is apparent that there was no significant decrease in $\mathrm{RBC}$ count between centrifugations. Due to the removal of plasma (the hematology analyser removes around 
$37050 \mu \mathrm{L}$ of the sample during each measurement) at every time instance, we noticed a slight increment in the RBC

371 count per litre of blood on some of the designs (and control). The absence of hemolysis in most of the samples

372 (see Section 3.4) corroborate this interpretation.

373 Secondly, a residual cell count can be used to establish the quality of the separated plasma. Figure 6.B shows

374 the remaining RBC count on the separated plasma. The remaining RBCs in design E2 was significantly lower

375 than that of the control at all time instances. Design E5 performed best at 3 minutes, however, due to the

376 vibrations reported earlier in this work, the separation reversed at 6 minutes. The best cell separation

377 performance was observed in the platelet counts (Figure 6.C). All adaptors (apart from E5) were capable of

378 separating more platelets than the control because of their much higher speed. Notably, design E2 separated

379 almost 4 times more platelets than the control, which could be of benefit in coagulation studies requiring

380 platelet-poor plasma. As explained in Materials and Methods, the separation performance was investigated on

381 pooled blood samples (see Materials and Method section), which has the advantage of removing individual sample specificities and enables accurate comparison between designs and control. To investigate any notable differences between individual pools, we compared the separation performance of designs E0, E2 and control after 10 minutes of centrifugation with two different pools of blood (Figure 6.D). The two pools investigated were found to have significant differences in original Hct (Pool 1, Hct $\approx 45.5 \%$; Pool 2, Hct $\approx 43.2 \%$ ). However, in terms of relative difference post and pre-centrifugation, the pools showed no significant differences in RBC and platelet relative counts.
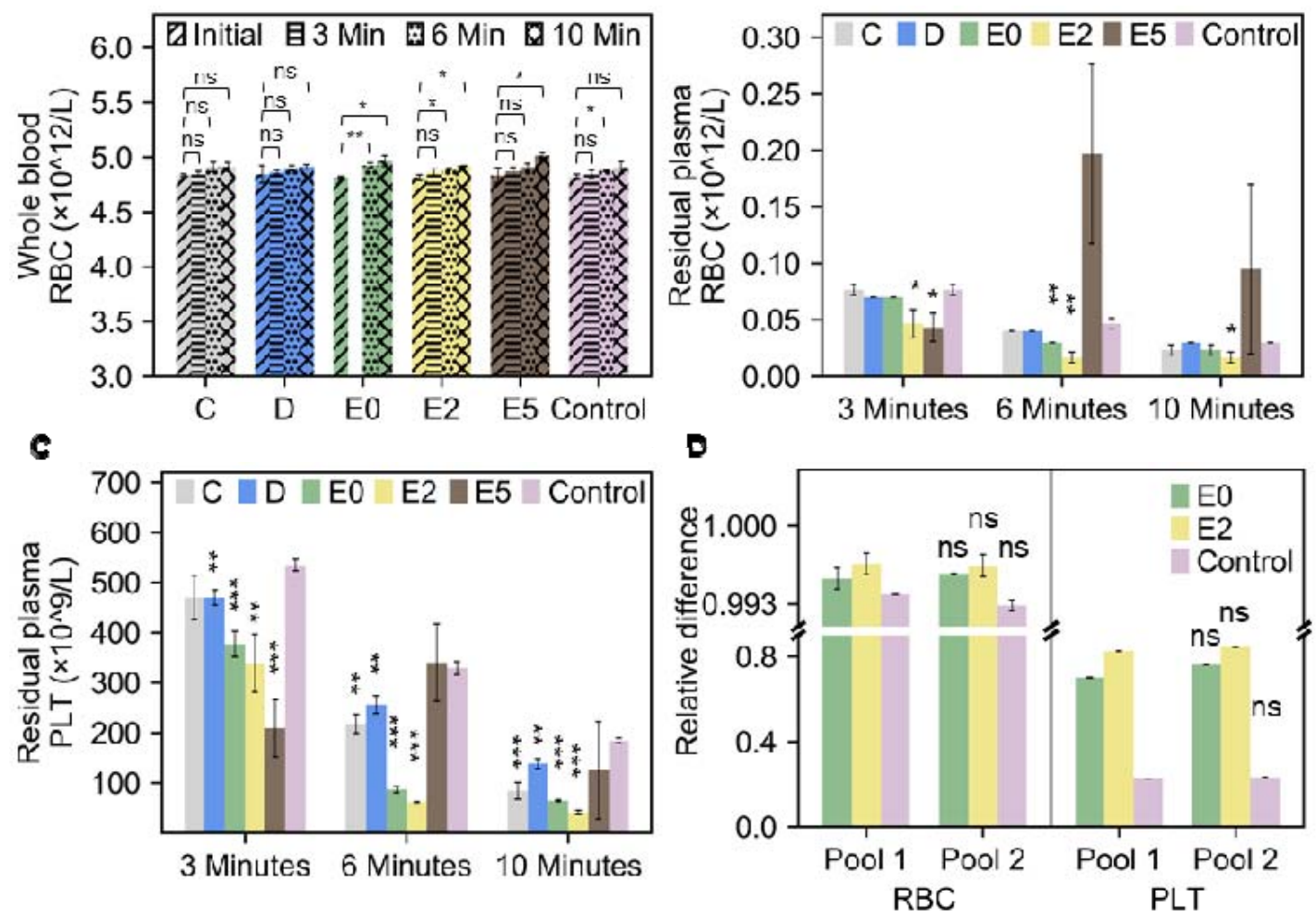
Figure 6: Blood count at 3, 6 and 10 minutes $(\boldsymbol{A})$ The red blood cell counts of pre (initial) and post (3,6, 10 minutes) separated blood show the integrity of the sample after centrifugation. The statistical indications relate to a non-paired t-test between the RBC counts between 0, 3, 6, and 10 minutes (B) The red blood cell (RBC) count on plasma after centrifugation shows RBC concentration with design E2 was significantly lower than the control in all periods. On the contrary, design E5, although performing well at 3 minutes was hampered by vibrations reversing the separation process. Here the statistics relate to a non-paired t-test between each of the designs and the control, for each time point (C) Platelet count (PLT) on separated plasma. Design E2 was able to separate almost 4 times higher platelet than the control within 10 minutes. Here the statistics relate to a nonpaired t-test between each of the designs and the control for each time point $(\boldsymbol{D})$ Relative RBC and Platelet counts after 10 minutes centrifugation from two different pools of blood. Here the statistics relate to a nonpaired t-test between Pool 1 and Pool 2 for each experiment. No significant difference was observed between different pools of blood.

\subsection{Plasma quality: Hemolysis detection}

To assess the quality of the separated samples, we measured the free-hemoglobin released after centrifugation and derived a hemolysis percentage (See Materials and Methods). Hgb levels from collected plasma samples of design E0, E2 and control (single and double centrifugation) were measured using Cripps method (See Materials and Methods). As reported in Figure 7A, all the measured samples had an estimated percentage of hemolysis between 1 to $1.7 \%$ corresponding to a free Hgb concentration of 0.2-0.4 g/dL. Despite the deflections observed on Designs E0 and E2, the separated plasma is well below the threshold of hemolysis limit and similar to the control used in our experiment, which shows the adapted device is adequate for general low-speed spin of clinical blood samples.

\subsection{Plasma quality: Protein load}

Albumin is known to be the most abundant plasma protein in humans which accounts for almost $60 \%$ of the total serum protein. It is an established biomarker of nutritional status [35] and reliable prognostic indicator for heart failure [36], pulmonary arterial hypertension [37], in acute surgical patients [38], morbidity and mortality [39] and many more diseases. The recovered plasma from the best designs (E0, and E2) and control centrifugation (single and double) were analysed to quantify the concentration of albumin. The albumin quantification from the sample was obtained by directly measuring the absorbance at $610 \mathrm{~nm}$ when the available albumin in a sample provides a peak in the presence of reagent bromocresol purple (see Materials and Methods). As shown in Figure 7.B, E0 and E2 samples (single and double centrifugation) showed a similar concentration to the full-scale centrifuge control, around $1.5 \mathrm{mM}(\sim 2.5 \mathrm{~g} / \mathrm{dL})$ with no statistically significant difference which shows the adapted centrifuge is suitable for plasma protein studies. The albumin concentration in all samples, including control samples, was slightly lower than the normal range of Albumin in plasma (3.4 to $5.4 \mathrm{~g} / \mathrm{dL})$, which can be explained by the age of the samples (3 days old).

\subsection{Plasma quality: cfDNA extraction}

Cell-free DNA (cfDNA) are small (50-200bp) DNA fragments that originate from cell apoptosis or necrosis. cfDNA overall levels or specific cfDNA regions can be used biomarkers in the diagnosis, prognosis, or monitoring of fetal chromosomal abnormalities, various cancers, infectious diseases and organ transplants [40]. 
430 This is an emerging biomarker that is now routinely and increasingly used in clinical practice. To assess the 431 suitability of the adapted centrifuge for cfDNA-based assays, we measured the levels of cfDNA using a LINE 432 PCR. Total cfDNA was extracted from $3 \mathrm{~mL}$ of separated plasma as per Material and Methods. After the first 433 centrifugation, we found the cfDNA concentration from design E0 and E2 to be higher (6.5-8 ng/ $\mu \mathrm{L})$ compared 434 to the control $(\sim 5 \mathrm{ng} / \mu \mathrm{L})$ (Figure $7 \mathrm{C}$ ). This could be due to the substandard pelleting of white blood cells singe 435 significantly more residual white blood cells (WBCs) were found in the adapted centrifugation, compared to the 436 control (Supplementary Figure S3). The lysis of remaining cells (including WBCs) during the cfDNA 437 extraction has introduced genomic DNA, resulting in overall higher cfDNA levels. Other studies also have 438 reported higher cfDNA concentration after single centrifugation [15]. A second, higher speed centrifugation is 439 often incorporated in cfDNA extraction protocols. This second spin at a higher speed (12,000×g RCF for 10 440 minutes), can be performed on smaller sample volumes using cheaper and smaller bench centrifuge. To test if 441 cfDNA yields can be brought to the same level as the control, we applied a second spin to both design E2 and 442 control samples. After this second centrifugation, both sample types showed a similar lower concentration of 443 cfDNA with no statistically significant difference. Generally speaking, it can be noted that the total cfDNA 444 levels are higher than levels reported elsewhere, this is due to age of the samples (three days old) which is sub445 optimal for cfDNA-based diagnostic, but adequate to characterise the performance of a device. Although 446 designs E0 and E2 showed similar RBCs separation and the yield compared to that of control, the low rotational 447 angle of $25^{\circ}$ resulted in lower buffy coat compaction and consequently higher WBC counts in the separated 448 plasma, compared to the benchmark. This issue might be addressed by exploring the use of a higher rotational 449 angle or longer spin duration. In the meantime, a second high-speed centrifugation can remove remaining WBCs 450 and lead to similar cfDNA levels in the adapted design and benchmark. 

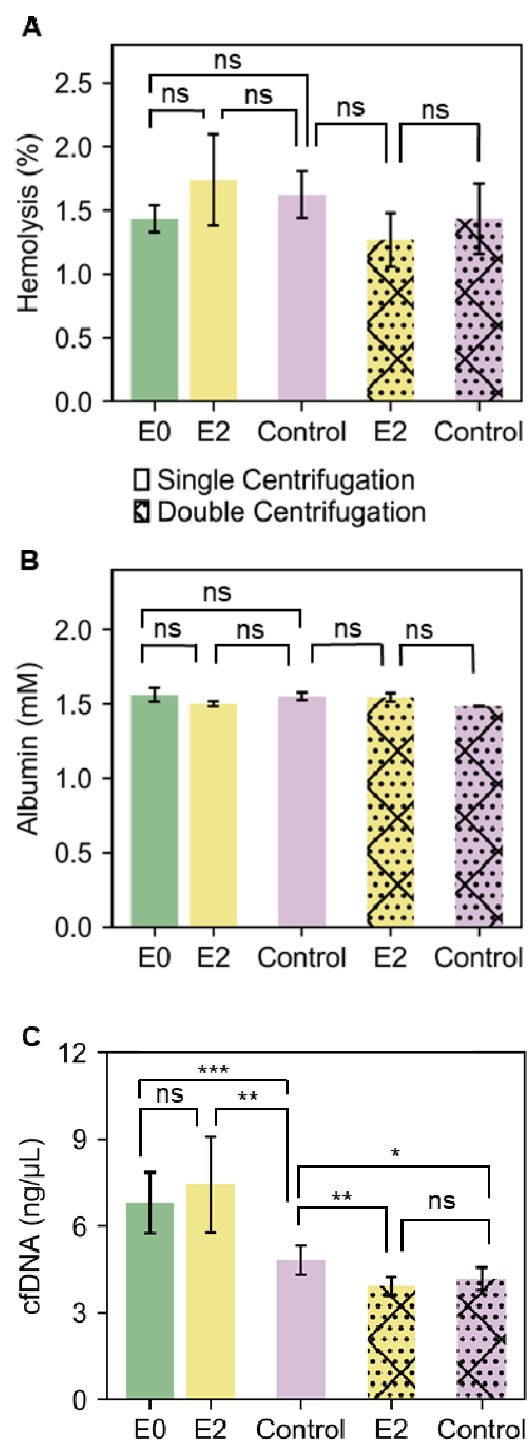

Figure 7: (A) Percentage of hemolysis in separated plasma from EO, E2 and control. (B) Albumin concentration in E0, E2 and control (C) Cell-free DNA levels in E0, E2, and control after single centrifugation or with additional second separate centrifugation

\section{Conclusion}

In this study, a mini-centrifuge costing $<\$ 130$ was adapted to handle large volume $(9 \mathrm{~mL})$ standard clinical samples in S-Monovette collection tubes. Our results showed that the air resistance was the most crucial parameter needed to be optimised to ensure an adequate centrifugal force for comparable cell separation to a commercial centrifuge. Following optimisation, the final design reached a speed of around 6725 RPM and RCF of 1060×g. Similar yield, cell counts (red blood cells, platelets), hemolysis and albumin levels were obtained from the optimised design and benchmark. Only the total cfDNA levels of the plasma separated in the adapted design were found to be significantly different to the benchmark, owing to a reduced separation performance for white blood cells. The nearest cheapest option for $9 \mathrm{~mL} \mathrm{S-Monovette} \mathrm{centrifugation} \mathrm{cost} \mathrm{over} \$ 1,500$. The bill of material for our adapted centrifuge stands at around $\$ 140$. The overall performance of the optimised adapted centrifuge, which costs a fraction of the total price of the commercial control centrifuge, was equivalent to a 
commercial centrifuge and superior to microfluidic approaches in yield, throughput and quality. Therefore, our design offers value and performance to the low-resource environment or could be further adapted to created portable diagnostic laboratories.

\section{References:}

[1] S. S. Sule, A. L. Petsiuk, and J. M. Pearce, "Open Source Completely 3-D Printable Centrifuge," Instruments, vol. 3, no. 2, p. 30, 2019.

[2] W. G. Pitt et al., "Rapid separation of bacteria from blood-review and outlook," Biotechnol. Prog., vol. 32, no. 4, pp. 823-839, Jul. 2016.

[3] H. Lee, W. Na, C. Park, K. H. Park, and S. Shin, "Centrifugation-free extraction of circulating nucleic acids using immiscible liquid under vacuum pressure," vol. 8, p. 5467, 2018.

[4] Z. Berk, "Centrifugation," in Food Process Engineering and Technology, Elsevier, 2013, pp. 241-257.

[5] A. Tsanni, "African scientists leverage open hardware," Nature, vol. 582, no. 7810, p. 138, Jun. 2020.

[6] M. Zastrow, "Open science takes on the coronavirus pandemic," Nature, vol. 581, no. 7806, pp. 109110, May 2020.

[7] A. Maia Chagas, L. L. Prieto-Godino, A. B. Arrenberg, and T. Baden, "The $€ 100$ lab: A 3D-printable open-source platform for fluorescence microscopy, optogenetics, and accurate temperature control during behaviour of zebrafish, Drosophila, and Caenorhabditis elegans," PLoS Biol., vol. 15, no. 7, Jul. 2017.

[8] A. K. Patel et al., "Design and Development of Low-cost Portable Centrifuge using Additive Manufacturing," 2019.

[9] M. S. Bhamla, B. Benson, C. Chai, G. Katsikis, A. Johri, and M. Prakash, "Hand-powered ultralow-cost paper centrifuge," Nat. Biomed. Eng., vol. 1, no. 1, 2017.

[10] C. H. Liu et al., "Blood Plasma Separation Using a Fidget-Spinner," Anal. Chem., vol. 91, no. 2, pp. 1247-1253, 2019.

[11] "How To Build Your Own DIY Centrifuge.".

[12] J. Ford, T. Goldstein, S. Trahan, A. Neuwirth, K. Tatoris, and S. Decker, "A 3D-printed nasopharyngeal swab for COVID-19 diagnostic testing," 3D Print. Med. 2020 61, vol. 6, no. 1, pp. 1-7, Aug. 2020.

[13] S. Ravindran, "How DIY technologies are democratizing science," Nature, vol. 587, no. 7834, pp. 509511, Nov. 2020.

[14] S. Bhagia et al., "Critical review of FDM 3D printing of PLA biocomposites filled with biomass resources, characterization, biodegradability, upcycling and opportunities for biorefineries," Appl. Mater. Today, vol. 24, p. 101078, Sep. 2021. 
[15] R. M. Trigg, L. J. Martinson, S. Parpart-Li, and J. A. Shaw, "Factors that influence quality and yield of circulating-free DNA: A systematic review of the methodology literature," Heliyon, vol. 4, no. 7, pp. e00699-e00699, Jul. 2018.

[16] Ultimaker, "Ultimaker Cura: Powerful, easy-to-use 3D printing software," Ultimaker, 2020. .

[17] L. B. Leverett, J. D. Hellums, C. P. Alfrey, and E. C. Lynch, "Red Blood Cell Damage by Shear Stress," Biophys. J., 1972.

[18] J. Fischer and J. Strackeljan, "Stability analysis of high speed lab centrifuges considering internal damping in rotor-shaft joints," 2006, pp. $131-147$.

[19] G. Lowe et al., "Nursing Blood Specimen Collection Techniques and Hemolysis Rates in an Emergency Department: Analysis of Venipuncture Versus Intravenous Catheter Collection Techniques," J. Emerg. Nurs., vol. 34, no. 1, pp. 26-32, Feb. 2008.

[20] R. Cardigan, "Guide to the Preparation, Use and Quality Assurance of Blood Components (13th edn)," Transfus. Med., vol. 17, no. 6, pp. 491-491, Dec. 2007.

[21] C. M. Cripps, "Rapid method for the estimation of plasma haemoglobin levels.," J. Clin. Pathol., vol. 21, no. 1, pp. 110-112, Jan. 1968.

[22] S. O. Sowemimo-Coker, "Red blood cell hemolysis during processing," Transfus. Med. Rev., vol. 16, no. 1, pp. 46-60, 2002.

[23] B. S et al., "Direct quantification of cell-free, circulating DNA from unpurified plasma," PLoS One, vol. 9, no. 3, Mar. 2014.

[24] F. Malizia, T. van Druenen, and B. Blocken, "Impact of wheel rotation on the aerodynamic drag of a time trial cyclist," Sport. Eng., vol. 24, no. 1, p. 3, 2021.

[25] S. F. Hoerner, Fluid Dynamic Drag: Practical Information on Aerodynamic Drag and Hydrodynamic Resistance, 2nd ed. New Jersey: Hoerner Fluid Dynamics, 1965.

[26] W. E. (Wilfred E. Baker, Explosion hazards and evaluation, 1st Editio. Elsevier Scientific Pub. Co, 1983.

[27] R. M. Nerem, "Vibration-Induced Arterial Shear Stress," Arch. Environ. Heal. An Int. J., vol. 26, no. 3, pp. 105-110, Mar. 1973.

[28] H. Ando, K. Nieminen, E. Toppila, J. Starck, and T. Ishitake, "Effect of impulse vibration on red blood cells in vitro," Scand. J. Work. Environ. Heal., vol. 31, no. 4, 2005.

[29] T. Jing, Y. Cheng, F. Wang, W. Bao, and L. Zhou, "Numerical investigation of centrifugal blood pump cavitation characteristics with variable speed," Processes, vol. 8, no. 3, 2020.

[30] M. Kersaudy-Kerhoas, D. M. Kavanagh, R. S. Dhariwal, C. J. Campbell, and M. P. Y. Desmulliez, "Validation of a blood plasma separation system by biomarker detection," Lab Chip, vol. 10, no. 12, pp. 
1587-1595, Jun. 2010.

[31] S. Tripathi, Y. V. B. Kumar, A. Agrawal, A. Prabhakar, and S. S. Joshi, "Microdevice for plasma separation from whole human blood using bio-physical and geometrical effects," Sci. Rep., vol. 6, 2016.

[32] M. Faivre, M. Abkarian, K. Bickraj, and H. A. Stone, "Geometrical focusing of cells in a microfluidic device: An approach to separate blood plasma," Biorheology, vol. 43, no. 2, pp. 147-159, 2006.

[33] E. Sollier, M. Cubizolles, Y. Fouillet, and J. L. Achard, "Fast and continuous plasma extraction from whole human blood based on expanding cell-free layer devices," Biomed. Microdevices, vol. 12, no. 3 , pp. 485-497, Jun. 2010.

[34] M. E. Haque, A. Matin, X. Wang, and M. Kersaudy-Kerhoas, "Effects of Syringe Pump Fluctuations On Cell-Free Layer in Hydrodynamic Separation Microfluidic Devices," May 2021.

[35] T. Hirata et al., "Associations of cardiovascular biomarkers and plasma albumin with exceptional survival to the highest ages," Nat. Commun., vol. 11, no. 1, p. 3820, 2020.

[36] W. Su et al., "Serum albumin is a useful prognostic indicator and adds important information to NTproBNP in a Chinese cohort of heart failure," Clin. Biochem., vol. 45, no. 7, pp. 561-565, 2012.

[37] D. Snipelisky, J. Jentzer, O. Batal, Z. Dardari, and M. Mathier, "Serum albumin concentration as an independent prognostic indicator in patients with pulmonary arterial hypertension," Clin. Cardiol., vol. 41, no. 6, pp. 782-787, Jun. 2018.

[38] K. SP, T. GJ, W. CW, and L. WY, "Serum albumin concentration as a prognostic indicator for acute surgical patients," Zhonghua Yi Xие Za Zhi (Taipei)., vol. 62, no. 2, pp. 61-67, Feb. 1999.

[39] L. YC et al., "Preoperative serum albumin level is a prognostic indicator for adenocarcinoma of the gastric cardia," J. Gastrointest. Surg., vol. 8, no. 8, pp. 1041-1048, Dec. 2004.

[40] A. J. Bronkhorst, V. Ungerer, and S. Holdenrieder, "The emerging role of cell-free DNA as a molecular marker for cancer management," Biomolecular Detection and Quantification, vol. 17. 2019. 